International Journal of Applied Mathematics

Volume 29 No. 4 2016, 451-464

ISSN: 1311-1728 (printed version); ISSN: 1314-8060 (on-line version)

doi: http://dx.doi.org/10.12732/ijam.v29i4.4

\title{
QUENCHING FOR A SEMILINEAR HEAT EQUATION WITH A SINGULAR BOUNDARY OUTFLUX
}

\author{
Burhan Selcuk $^{1 \S}$, Nuri Ozalp ${ }^{2}$ \\ ${ }^{1}$ Department of Computer Engineering \\ Karabuk University, Balıklar Mevki \\ 78050 Karabuk, TURKEY \\ ${ }^{2}$ Department of Mathematics \\ Ankara University \\ 06100 Ankara, TURKEY
}

\begin{abstract}
In this paper, we study the quenching behavior of solution of a semilinear heat equation with a singular boundary outflux. We first get a local existence result for this problem. We prove finite time quenching for the solution, we show that quenching occurs on the boundary and the time derivative blows up at the quenching time under certain conditions. Finally, we get a quenching rate and a lower bound for quenching time.
\end{abstract}

AMS Subject Classification: 35K20, 35K55, 35B50

Key Words: semilinear heat equation, singular boundary outflux, quenching, maximum principles, monotone iterations

\section{Introduction}

We consider the following quenching behavior of a semilinear heat equation with a singular boundary outflux:

$$
\left\{\begin{array}{l}
u_{t}=u_{x x}+(1-u)^{-\alpha}, \quad 0<x<1,0<t<T \\
u_{x}(0, t)=0, u_{x}(1, t)=-u^{-\beta}(1, t), \quad 0<t<T, \\
u(x, 0)=u_{0}(x), \quad 0 \leq x \leq 1,
\end{array}\right.
$$

where $\alpha, \beta$ are positive constants and $T(\leq \infty)$ is the quenching time. The

Received: June 1, 2016

(C) 2016 Academic Publications

${ }^{\S}$ Correspondence author 
initial function $u_{0}:[0,1] \rightarrow(0,1)$ satisfies the compatibility conditions

$$
u_{0}^{\prime}(0)=0, u_{0}^{\prime}(1)=-u_{0}^{-\beta}(1) .
$$

Our main purpose is to examine the quenching behavior of the solution of the problem (1.1) having different singular heat sources.

Definition 1. A solution $u(x, t)$ of problem (1.1) is said to quench if there exists a finite time $T$ such that

$$
\begin{gathered}
\lim _{t \rightarrow T^{-}} \max \{u(x, t): 0 \leq x \leq 1\} \rightarrow 1, \quad \text { or } \\
\lim _{t \rightarrow T^{-}} \min \{u(x, t): 0 \leq x \leq 1\} \rightarrow 0 .
\end{gathered}
$$

In [8], Selcuk and Ozalp considered the problem (1.1). They showed that $x=0$ is the quenching point in finite time, $\lim _{t \rightarrow T^{-}} u(0, t) \rightarrow 1$, if $u_{0}$ satisfies $u_{x x}(x, 0)+(1-u(x, 0))^{-\alpha} \geq 0$ and $u_{x}(x, 0) \leq 0$. Further they showed that $u_{t}$ blows up at quenching time. Furthermore, they obtained a quenching rate and a lower bound for quenching time.

Throughout this paper, we assume that the initial function $u_{0}$ satisfies

$$
\begin{aligned}
u_{x x}(x, 0)+(1-u(x, 0))^{-\alpha} & \leq 0 \\
u_{x}(x, 0) & \leq 0 .
\end{aligned}
$$

Since 1975, quenching problems with various boundary conditions have been studied extensively ([1] - [4] and [6] - [9]). To mention some, Fila and Levine [3] considered a heat equation with a singular boundary outflux:

$$
\left\{\begin{array}{l}
u_{t}=u_{x x}, 0<x<1,0<t<T \\
u_{x}(0, t)=0, u_{x}(1, t)=-u^{-\beta}(1, t), 0<t<T \\
u(x, 0)=u_{0}(x)>0,0 \leq x \leq 1
\end{array}\right.
$$

They showed that $x=1$ is the unique quenching point in finite time, under certain hypotheses on $u_{0}$. Further, they obtained a lower bound for quenching time $T, T \geq u_{0}^{2(\beta+1)}(1) /(2 \beta(\beta+1))$. Furthermore, they obtained the quenching 
rate estimate which is $(T-t)^{1 / 2(\beta+1)}$. Deng and $\mathrm{Xu}[2]$ considered a problem with nonlinear boundary outflux at one side:

$$
\left\{\begin{array}{l}
\left(u^{m}\right)_{t}=u_{x x}, 0<x<1,0<t<T, \\
u_{x}(0, t)=0, u_{x}(1, t)=-u^{-\beta}(1, t), 0<t<T, \\
u(x, 0)=u_{0}(x)>0,0 \leq x \leq 1,
\end{array}\right.
$$

where $0<\beta, m<\infty$. They showed that $u$ quenches in finite time $T$ and the only quenching point is $x=1$. Further, they also gave the quenching rate estimate which is $(T-t)^{1 /(m+2 \beta+1)}$. In [9], Zhi and Mu considered a problem with nonlinear boundary outflux at one side:

$$
\left\{\begin{array}{l}
u_{t}=u_{x x}+(1-u)^{-\alpha}, 0<x<1,0<t<T \\
u_{x}(0, t)=u^{-\beta}(1, t), u_{x}(1, t)=0,0<t<T \\
u(x, 0)=u_{0}(x)>0,0 \leq x \leq 1
\end{array}\right.
$$

where $\alpha, \beta>0$. They showed that $u$ quenches in finite time $T$ and the only quenching point is $x=0$. Further, they also gave the quenching rate estimate which is $(T-t)^{1 / 2(\beta+1)}$.

So far in literature, quenching problem with different type of two singular sources, which one is reaction term the other is absorption term is studied in a few. The quenching problem (1.1) with two type of singularity terms, namely, a reaction term $(1-u)^{-\alpha}$ and the boundary outflux term $-u^{-\beta}$. As in [8], observe that in problem (1.1) the singular source term may become singular if $u(x, t) \rightarrow 1^{-}$as $(x, t) \rightarrow\left(x^{*}, T\right)$, where $x^{*}$ is a quenching point in $[0,1]$ and $T$ is a quenching time in $(0, \infty)$. On the other hand, the outflux $-u^{-\beta}(1, t)$ may also become singular in some finite time (see [2], [3] and [9]). Here, we discuss the second situation. This paper is arranged as follows. In Section 2, we firstly obtain a local existence result for problem (1.1). In Section 3, we show that quenching occurs in finite time, the only quenching point is $x=1$ and $u_{t}$ blows up at quenching time under conditions (1.2) - (1.3). Finally, we get a quenching rate and a lower bound for quenching time.

\section{Local Existence}

It is well known that one of the most effective methods to obtain existence and uniqueness results of the solution of parabolic equations with initial conditions is monotone iterative technique (for details see [1] and [7]).

Let $C^{m}(Q), C^{\alpha}(Q)$ be the respective spaces of $m$-times differentiable and Hölder continuous functions in $Q$ with exponent $\alpha \in(0,1)$, where $Q$ is any domain. Denote by $C^{2,1}([0,1] \times[0, T))$ the set of functions that are twice 
continuously differentiable in $x$ and once continuously differentiable in $t$ for $(x, t) \in[0,1] \times[0, T)$. It assumed that initial function $u_{0}(x)$ is in $C^{2+\alpha}$.

Definition 2. $\widetilde{u}$ is called an upper solution of the problem (1.1) if $\widetilde{u} \in$ $C([0,1] \times[0, T)) \cap C^{2,1}((0,1) \times(0, T))$ and $\widetilde{u}$ satisfies the following conditions:

$$
\begin{aligned}
& \widetilde{u}_{t}-\widetilde{u}_{x x} \geq(1-\widetilde{u})^{-\alpha}, 0<x<1,0<t<T, \\
& \widetilde{u}_{x}(0, t)=0, \widetilde{u}_{x}(1, t) \geq-\widetilde{u}^{-\beta}(1, t), 0<t<T, \\
& \widetilde{u}(x, 0) \geq u_{0}(x), 0 \leq x \leq 1 .
\end{aligned}
$$

The lower solution of problem (1.1), $\widehat{u} \in C([0,1] \times[0, T)) \cap C^{2,1}((0,1) \times(0, T))$ is defined by reversing the inequalities.

Lemma 3. Let $\widetilde{u}$ and $\widehat{u}$ be a positive upper solution and a nonnegative lower solution of problem $(1.1)$ in $[0,1] \times[0, T)$, respectively. Then, we get the following results:

(a) $\widetilde{u} \geq \widehat{u}$ in $[0,1] \times[0, T)$,

(b) if $u^{*}$ is a solution, then $\widetilde{u} \geq u^{*} \geq \widehat{u}$ in $[0,1] \times[0, T)$.

Proof. We give the proof by utilizing Lemma 3.1 in [5]. Let $v(x, t)=\widetilde{u}-\widehat{u}$. Then $v(x, t)$ satisfies

$$
\begin{aligned}
& v_{t} \geq v_{x x}+\alpha(1-\eta)^{-\alpha-1} v, 0<x<1,0<t<T, \\
& v_{x}(0, t)=0, v_{x}(1, t) \geq \beta \xi^{-\beta-1}(1, t) v(1, t), 0<t<T, \\
& v(x, 0) \geq 0,0 \leq x \leq 1,
\end{aligned}
$$

where $\eta(x, t)$ lies between $\widetilde{u}(x, t)$ and $\widehat{u}(x, t)$ and $\xi(1, t)$ lies between $\widetilde{u}(1, t)$ and $\widehat{u}(1, t)$. For any fixed $\tau \in(0, T)$, let

$$
\begin{aligned}
L & =\max _{0 \leq x \leq 1,0 \leq t \leq \tau}\left(\frac{1}{2} \beta \xi^{-\beta-1}(x, t)\right) \\
M & =2 L+4 L^{2}+\max _{0 \leq x \leq 1,0 \leq t \leq \tau}\left(\alpha(1-\eta(x, t))^{-\alpha-1}\right) .
\end{aligned}
$$

Set $w(x, t)=e^{-M t-L x^{2}} v(x, t)$. Then $w$ satisfies

$$
\begin{aligned}
& w_{t} \geq w_{x x}+4 L x w_{x}+c w, 0<x<1,0<t \leq \tau, \\
& w_{x}(0, t)=0, w_{x}(1, t) \geq d(t) w(1, t), 0<t \leq \tau \\
& w(x, 0) \geq 0,0 \leq x \leq 1
\end{aligned}
$$

where $c=c(x, t) \leq 0$ and $d=d(t) \leq 0$. By the maximum principle, we obtain that $w \geq 0$ in $[0,1] \times[0, \tau]$. Thus, $\widetilde{u} \geq \widehat{u}$ in $[0,1] \times[0, T)$. 
(b) It is clear from Definition 2 that every solution of the problem (1.1) is an upper solution as well as a lower solution of the corresponding problem. If $u^{*}$ is a solution, then we get

$$
\widetilde{u} \geq u^{*} \text { and } u^{*} \geq \widehat{u}
$$

and

$$
\widetilde{u} \geq u^{*} \geq \widehat{u}
$$

in $[0,1] \times[0, T)$ from Lemma 3 -(a).

For a given pair of ordered upper and lower solutions $\widetilde{u}$ and $\widehat{u}$ we set

$$
S=\{u \in C([0,1] \times[0, T)): \widehat{u} \leq u \leq \widetilde{u}\} .
$$

Let

$$
f(x, t, u(x, t))=(1-u(x, t))^{-\alpha}, g(x, t, u(x, t))=-u^{-\beta}(x, t) .
$$

Throughout this section, we make the following hypothesis on the above functions in problem (1.1):

$\left(H_{1}\right)$-(i) The functions $f(x, t,$.$) is in C^{\alpha, \alpha / 2}([0,1] \times[0, T))$ and $g(x, t,$.$) is in$ $C^{1+\alpha,(1+\alpha) / 2}(\{1\} \times(0, T))$, respectively.

$\left(H_{1}\right)$-(ii) Let $f(., u)$ and $g(., u)$ are $C^{1}$-functions of $u$ for $u \in S$. Also,

$$
\begin{gathered}
f_{u}(x, t, u) \geq 0 \text { for } u \in S,(x, t) \in[0,1] \times[0, T), \\
g_{u}(x, t, u) \geq 0 \text { for } u \in S,(x, t) \in\{1\} \times(0, T) .
\end{gathered}
$$

The condition (2.1) implies that $f(., u), g(., u)$ are non-decreasing in $u$, which is crucial for the construction of monotone sequences.

Next, we are going to construct monotone sequences of functions which give the estimation of the solution $u$ of problem (1.1). Specifically, by starting from any initial iteration $u^{0}$ we can construct a sequence $\left\{u^{(k)}\right\}$ from the linear iteration process

$$
\left\{\begin{array}{l}
u_{t}^{(k)}-u_{x x}^{(k)}=f\left(x, t, u^{(k-1)}\right), \quad 0<x<1,0<t<T \\
u_{x}^{(k)}(0, t)=0, u_{x}^{(k)}(1, t)=g\left(1, t, u^{(k-1)}\right), \quad 0<t<T \\
u^{(k)}(x, 0)=u_{0}(x), \quad 0 \leq x \leq 1
\end{array}\right.
$$

It is clear that the sequence governed by (2.2) is well defined and can be obtained by solving a linear initial boundary value problem. Starting from initial iteration $u^{0}=\widetilde{u}$ and $u^{0}=\widehat{u}$, we define two sequences of the functions $\left\{\bar{u}^{(k)}\right\}$ and $\left\{\underline{u}^{(k)}\right\}$ for $k=1,2, \ldots$ respectively, and refer to them as maximal and minimal sequences, respectively, where those functions satisfy the above linear problem. 
Lemma 4. The sequences $\left\{\bar{u}^{(k)}\right\},\left\{\underline{u}^{(k)}\right\}$ possess the monotone property

$$
\widehat{u} \leq \underline{u}^{(k)} \leq \underline{u}^{(k+1)} \leq \bar{u}^{(k+1)} \leq \bar{u}^{(k)} \leq \widetilde{u}
$$

for $(x, t) \in[0,1] \times[0, T)$ and every $k=1,2, \ldots$

Proof. Let $\mu=\widetilde{u}-\bar{u}^{(1)}$. From (2.2) and Definition 2, we get

$$
\begin{aligned}
& \mu_{t}-\mu_{x x}=\widetilde{u}_{t}-\widetilde{u}_{x x}-f(x, t, \widetilde{u}) \geq 0,0<x<1,0<t<T, \\
& \mu_{x}(0, t)=0, \mu_{x}(1, t)=\widetilde{u}_{x}(1, t)-g(1, t, \widetilde{u}) \geq 0, \quad 0<t<T, \\
& \mu(x, 0)=\widetilde{u}(x, 0)-u_{0}(x) \geq 0, \quad 0 \leq x \leq 1 .
\end{aligned}
$$

From Maximum principle and Hopf's Lemma for parabolic equations, we get $\mu \geq 0$ for $(x, t) \in[0,1] \times[0, T)$, i.e. $\bar{u}^{(1)} \leq \widetilde{u}$. Similarly, using the property of a lower solution, we obtain $\underline{u}^{(1)} \geq \widehat{u}$.

Let $\mu^{(1)}=\bar{u}^{(1)}-\underline{u}^{(1)}$. From $(2.1)$ and (2.2), we get

$$
\begin{aligned}
& \mu_{t}^{(1)}-\mu_{x x}^{(1)}=f(x, t, \widetilde{u})-f(x, t, \widehat{u}) \geq 0,0<x<1,0<t<T, \\
& \mu_{x}^{(1)}(0, t)=0, \mu_{x}^{(1)}(1, t)=g(1, t, \widetilde{u})-g(1, t, \widehat{u}) \geq 0,0<t<T, \\
& \mu^{(1)}(x, 0)=u_{0}(x)-u_{0}(x)=0,0 \leq x \leq 1 .
\end{aligned}
$$

From Maximum principle and Hopf's Lemma for parabolic equations, we get $\mu^{(1)} \geq 0$ for $(x, t) \in[0,1] \times[0, T)$, i.e. $\underline{u}^{(1)} \leq \bar{u}^{(1)}$. Therefore,

$$
\widehat{u} \leq \underline{u}^{(1)} \leq \bar{u}^{(1)} \leq \widetilde{u}
$$

for $(x, t) \in[0,1] \times[0, T)$.

Assume that

$$
\underline{u}^{(k-1)} \leq \underline{u}^{(k)} \leq \bar{u}^{(k)} \leq \bar{u}^{(k-1)}
$$

for $(x, t) \in[0,1] \times[0, T)$ and for some integer $k>1$. Let $\mu^{(k)}=\bar{u}^{(k)}-\bar{u}^{(k+1)}$. From (2.1) and (2.2), we get

$$
\begin{aligned}
& \mu_{t}^{(k)}-\mu_{x x}^{(k)}=f\left(x, t, \bar{u}^{(k-1)}\right)-f\left(x, t, \bar{u}^{(k)}\right) \geq 0,0<x<1,0<t<T, \\
& \mu_{x}^{(k)}(0, t)=0, \mu_{x}^{(k)}(1, t)=g\left(1, t, \bar{u}^{(k-1)}\right)-g\left(1, t, \bar{u}^{(k)}\right) \geq 0,0<t<T, \\
& \mu^{(k)}(x, 0)=0,0 \leq x \leq 1 .
\end{aligned}
$$

From Maximum principle and Hopf's Lemma for parabolic equations, we get $\mu^{(k)} \geq 0$ for $(x, t) \in[0,1] \times[0, T)$, i.e. $\bar{u}^{(k+1)} \leq \bar{u}^{(k)}$. A similar argument gives $\underline{u}^{(k+1)} \geq \underline{u}^{(k)}$ and $\bar{u}^{(k+1)} \geq \underline{u}^{(k+1)}$. Therefore, the lemma holds from the mathematical induction. 
Lemma 5. For each positive integer $k, \bar{u}^{(k)}$ is an upper solution, $\underline{u}^{(k)}$ is a lower solution, $\underline{u}^{(k)} \leq \bar{u}^{(k)}$ for $(x, t) \in[0,1] \times[0, T)$.

Proof. From (2.1), (2.2) and Lemma 3, $\bar{u}^{(k)}$ satisfies

$$
\begin{aligned}
& \bar{u}_{t}^{(k)}-\bar{u}_{x x}^{(k)}=f\left(x, t, \bar{u}^{(k-1)}\right)=f\left(x, t, \bar{u}^{(k-1)}\right)-f\left(x, t, \bar{u}^{(k)}\right)+f\left(x, t, \bar{u}^{(k)}\right) \\
& \geq f\left(x, t, \bar{u}^{(k)}\right), \\
& \bar{u}_{x}^{(k)}(0, t)=0, \bar{u}_{x}^{(k)}(1, t)=g\left(1, t, \bar{u}^{(k-1)}\right) \\
& =g\left(1, t, \bar{u}^{(k-1)}\right)-g\left(1, t, \bar{u}^{(k)}\right)+g\left(1, t, \bar{u}^{(k)}\right) \geq g\left(1, t, \bar{u}^{(k)}\right), \\
& \bar{u}^{(k)}(x, 0)=u_{0}(x), 0 \leq x \leq 1,
\end{aligned}
$$

and $\underline{u}^{(k)}$ satisfies

$$
\begin{aligned}
& \underline{u}_{t}^{(k)}-\underline{u}_{x x}^{(k)}=f\left(x, t, \underline{u}^{(k-1)}\right) \\
& =f\left(x, t, \underline{u}^{(k-1)}\right)-f\left(x, t, \underline{u}^{(k)}\right)+f\left(x, t, \underline{u}^{(k)}\right) \leq f\left(x, t, \underline{u}^{(k)}\right), \\
& \underline{u}_{x}^{(k)}(0, t)=0, \underline{u}_{x}^{(k)}(1, t)=g\left(1, t, \underline{u}^{(k-1)}\right) \\
& =g\left(1, t, \underline{u}^{(k-1)}\right)-g\left(1, t, \underline{u}^{(k)}\right)+g\left(1, t, \underline{u}^{(k)}\right) \leq g\left(1, t, \underline{u}^{(k)}\right), \\
& \underline{u}^{(k)}(x, 0)=u_{0}(x), 0 \leq x \leq 1 .
\end{aligned}
$$

From Lemma 4 and above inequalities, the functions $\bar{u}^{(k)}$ and $\underline{u}^{(k)}$ are ordered upper and lower solutions of problem (2.2).

We have the following existence theorem for problem (1.1) via Lemma 4 and Lemma 5.

Theorem 6. Let $\widetilde{u}, \widehat{u}$ be a pair of ordered upper and lower solutions of problem (1.1), and let Hypothesis $\left(H_{1}\right)$ hold. Then the sequences $\left\{\bar{u}^{(k)}\right\},\left\{\underline{u}^{(k)}\right\}$ given by the problem (2.2) with $u^{0}=\widetilde{u}$ and $u^{0}=\widehat{u}$ converge monotonically to a maximal solution $\bar{u}$ and minimal solution $\underline{u}$ of problem (1.1), respectively. Further,

$$
\widehat{u} \leq \underline{u}^{(k)} \leq \underline{u}^{(k+1)} \leq \underline{u} \leq \bar{u} \leq \bar{u}^{(k+1)} \leq \bar{u}^{(k)} \leq \widetilde{u}
$$

for $(x, t) \in[0,1] \times[0, T)$ and each positive integer $k$. Furthermore, if $\underline{u}=$ $\bar{u}\left(\equiv u^{*}\right)$, then $u^{*}$ is the unique solution of the problem (1.1) in $S$.

Proof. The pointwise limits

$$
\lim _{k \rightarrow \infty} \bar{u}^{(k)}(x, t)=\bar{u}(x, t), \lim _{k \rightarrow \infty} \underline{u}^{(k)}(x, t)=\underline{u}(x, t)
$$


exist and satisfy relation (2.3). Indeed, the sequence $\left\{\bar{u}^{(k)}\right\}$ is monotone nonincreasing which is bounded from below, while the sequence $\left\{\underline{u}^{(k)}\right\}$ is monotone nondecreasing and is bounded from Lemma 4.

Let $\Theta=\underline{u}(x, t)-\bar{u}(x, t)$. From (2.3), we have $\underline{u}(x, t) \leq \bar{u}(x, t)$ for $(x, t) \in$ $[0,1] \times[0, T)$. Also, then $\Theta(x, t)$ satisfies

$$
\begin{aligned}
& \Theta_{t}-\Theta_{x x}=f(x, t, \underline{u})-f(x, t, \bar{u}), 0<x<1,0<t<T, \\
& \Theta_{x}(0, t)=0,0<t<T, \\
& \Theta_{x}(1, t)=g(1, t, \underline{u})-g(1, t, \bar{u}), 0<t<T, \\
& \Theta(x, 0)=0,0 \leq x \leq 1 .
\end{aligned}
$$

By using Lemma 3-(a) and Lemma 1 in [8], $\Theta \geq 0$ for $(x, t) \in[0,1] \times[0, T)$, i.e. $\underline{u}(x, t) \geq \bar{u}(x, t)$. Then, we get $\underline{u}(x, t)=\bar{u}(x, t)$.

If $u^{*}$ is any other solution in $S$, then we get from Lemma 5 ,

$$
\bar{u} \geq u^{*} \text { and } u^{*} \geq \underline{u}
$$

and

$$
\bar{u} \geq u^{*} \geq \underline{u}
$$

in $[0,1] \times[0, T)$. This implies that

$$
\bar{u}=u^{*}=\underline{u}
$$

and hence $u^{*}$ is the unique solution of problem (1.1).

\section{Quenching on the Boundary and Blow-Up of $u_{t}$}

In this section, we investigate the quenching behavior of problem (1.1).

Remark 1. If $u_{0}$ satisfies (1.3), then we get $u_{x}<0$ in $(0,1] \times(0, T)$ by the maximum principle. Thus we get $u(0, t)=\max _{0 \leq x \leq 1} u(x, t)$.

Remark 2. If $u_{0}$ satisfies (1.2), then we get $u_{t}(x, t) \leq 0$ in $[0,1] \times[0, T)$ (we can give the proof similar to proof of Lemma 3.1 in [5] and Lemma 1 in $[8])$. Also, for any $(\xi, \eta) \in(0,1) \times(0, T)$, there exists a subset $\left[x_{1}, x_{2}\right] \times\left[t_{1}, t_{2}\right]$ of $(0,1) \times(0, T)$ such that $(\xi, \eta) \in\left[x_{1}, x_{2}\right] \times\left[t_{1}, t_{2}\right]$. Define, $H=u_{t}$ in $\left[x_{1}, x_{2}\right] \times$ $\left[t_{1}, t_{2}\right]$. We get

$$
\begin{aligned}
H_{t}-H_{x x} & =\alpha(1-u)^{-\alpha-1} H \text { in }\left(x_{1}, x_{2}\right) \times\left(t_{1}, t_{2}\right), \\
H & \leq 0 \text { on }\left[x_{1}, x_{2}\right] \times\left[t_{1}, t_{2}\right] .
\end{aligned}
$$


The strong maximum principle implies that either $H<0$ or $H \equiv 0$ in $\left(x_{1}, x_{2}\right) \times$ $\left(t_{1}, t_{2}\right)$. Since $H \equiv 0$ contradicts to the fact that $u(x, t)$ is strictly decreasing in $t, u_{t}<0$. Because $(\xi, \eta)$ is arbitrary in $(0,1) \times(0, T)$, we have $u_{t}<0$ in $(0,1) \times(0, T)$.

Theorem 7. If $u_{0}$ satisfies (1.2), then there exists a finite time $T$, such that the solution $u$ of problem (1.1) quenches at time $T$.

Proof. Assume that $u_{0}$ satisfies (1.2). Then we get

$$
\omega=u^{-q}(1,0)-\int_{0}^{1}(1-u(x, 0))^{-p} d x>0 .
$$

Introduce a mass function: $m(t)=\int_{0}^{1} u(x, t) d x, 0<t<T$. Then

$$
m^{\prime}(t)=-u^{-q}(1, t)+\int_{0}^{1}(1-u(x, t))^{-p} d x \leq-\omega,
$$

by Remark 2 . Thus, $m(t) \leq m(0)-\omega t$, which means that $m\left(T_{0}\right)=0$ for some $T_{0},\left(0<T \leq T_{0}\right)$ and so, $u$ quenches in a finite time.

Theorem 8. If $u_{0}$ satisfies (1.3), then $x=1$ is the only quenching point.

Proof. Define

$$
J(x, t)=u_{x}+\varepsilon\left(x-b_{1}\right) \text { in }\left[b_{1}, b_{2}\right] \times[\tau, T),
$$

where $b_{1} \in[0,1), b_{2} \in\left(b_{1}, 1\right], \tau \in(0, T)$ and $\varepsilon$ is a positive constant to be specified later. Then, $J(x, t)$ satisfies

$$
J_{t}-J_{x x}=\alpha(1-u)^{-\alpha-1} u_{x}<0 \text { in }\left(b_{1}, b_{2}\right) \times[\tau, T),
$$

since $u_{x}(x, t)<0$ in $[0,1] \times[0, T)$. Thus, $J(x, t)$ cannot attain a positive interior maximum by the maximum principle. Further, if $\varepsilon$ is small enough, $J(x, \tau)<0$ since $u_{x}(x, t)<0$ in $(0,1] \times[0, T)$. Furthermore, if $\varepsilon$ is small enough,

$$
\begin{aligned}
& J\left(b_{1}, t\right)=u_{x}\left(b_{1}, t\right)<0, \\
& J\left(b_{2}, t\right)=u_{x}\left(b_{2}, t\right)+\varepsilon\left(b_{2}-b_{1}\right)<0,
\end{aligned}
$$

for $t \in(\tau, T)$. By the maximum principle, we obtain that $J(x, t)<0$, i.e. $u_{x}<-\varepsilon\left(x-b_{1}\right)$ for $(x, t) \in\left[b_{1}, b_{2}\right] \times[\tau, T)$. Integrating this with respect to $x$ from $b_{1}$ to $b_{2}$, we have

$$
u\left(b_{2}, t\right)<u\left(b_{1}, t\right)-\frac{\varepsilon\left(b_{2}-b_{1}\right)^{2}}{2}
$$


and

$$
u\left(b_{1}, t\right)>u\left(b_{2}, t\right)+\frac{\varepsilon\left(b_{2}-b_{1}\right)^{2}}{2}>0 .
$$

So $u$ does not quench in $[0,1)$. The theorem is proved.

Theorem 9. If $u_{0}$ satisfies (1.2) - (1.3), then $u_{t}$ blows up at the quenching time.

Proof. Define

$$
J(x, t)=u_{t}+\varepsilon\left(x-b_{1}\right) u^{-\beta} \text { in }\left[b_{1}, b_{2}\right] \times[\tau, T),
$$

where $b_{1} \in[0,1), b_{2} \in\left(b_{1}, 1\right], \tau \in(0, T)$ and $\varepsilon$ is a positive constant to be specified later. Then, $J(x, t)$ satisfies

$$
\begin{aligned}
J_{t}-J_{x x}-\alpha(1-u)^{-\alpha-1} J= & -\varepsilon\left(x-b_{1}\right)(1-u)^{-\alpha} u^{-\beta}\left[\beta u^{-1}+\alpha(1-u)^{-1}\right] \\
& +2 \varepsilon u^{-\beta-1} u_{x}-\varepsilon \beta(\beta+1)\left(x-b_{1}\right) u^{-\beta-2} u_{x}^{2} \\
< & 0
\end{aligned}
$$

in $(0,1) \times[0, T) . J(x, \tau) \leq 0$ if $\varepsilon$ is small enough and by Remark 2. Further, if $\varepsilon$ is small enough,

$$
\begin{aligned}
& J\left(b_{1}, t\right)=u_{t}\left(b_{1}, t\right)<0, \\
& J\left(b_{2}, t\right)=u_{t}\left(b_{2}, t\right)+\varepsilon\left(b_{2}-b_{1}\right) u^{-\beta}<0,
\end{aligned}
$$

for $t \in(\tau, T)$. By the maximum principle and Hopf lemma, we obtain that $J(x, t) \leq 0$ for $(x, t) \in\left[b_{1}, b_{2}\right] \times[0, T)$. Namely, $u_{t} \leq-\varepsilon\left(x-b_{1}\right) u^{-\beta}(x, t)$ for $(x, t) \in\left[b_{1}, b_{2}\right] \times[\tau, T)$, i.e. $u_{t} \leq-\varepsilon x u^{-\beta}(x, t)$ for $(x, t) \in[0,1] \times[\tau, T)$. For $x=1$, we get

$$
u_{t}(1, t) \leq-\varepsilon u^{-\beta}(1, t),
$$

and

$$
\lim _{t \rightarrow T^{-}} u_{t}(1, t) \leq \lim _{t \rightarrow T^{-}}-\varepsilon u^{-\beta}(1, t)=-\infty .
$$

The theorem is proved. 


\section{Quenching Rate}

In this section, we get a quenching rate for problem (1.1). For this, we assume that

$$
u_{x}(x, 0) \leq-x u^{-\beta}(x, 0), 0 \leq x \leq 1 .
$$

Theorem 10. If $u_{0}$ satisfies $(1.2)-(1.3)$, then there exists a positive constant $C_{1}$ such that

$$
u(1, t) \geq C_{1}(T-t)^{1 / 2(\beta+1)},
$$

for $t$ sufficiently close to $T$.

Proof. Define $M(x, t)=u_{t}-\delta \beta u^{-\beta-1} u_{x}$ in $[0,1] \times[\tau, T)$, where $\tau \in(0, T)$ and $\delta$ is a positive constant to be specified later. Then, $M(x, t)$ satisfies

$$
\begin{gathered}
M_{t}-M_{x x}-\alpha(1-u)^{-\alpha-1} M \\
=\delta \beta(\beta+1) u^{-\beta-2} u_{x}\left[-2 u_{t}+3(1-u)^{-\alpha}+(\beta+2) u^{-1} u_{x}^{2}\right]<0,
\end{gathered}
$$

for $(x, t) \in(0,1) \times(\tau, T)$, since $u_{t}<0$ and $u_{x}<0$ in $(0,1) \times(0, T)$. Further, if $\delta$ is small enough, $M(x, \tau) \leq 0$ for $x \in[0,1]$, and $M(0, t) \leq 0, M(1, t)<0$ for $t \in[\tau, T)$. By the maximum principle, we obtain that $M(x, t) \leq 0$ for $(x, t) \in[0,1] \times[\tau, T)$. Namely, $u_{t} \leq \delta \beta u^{-\beta-1} u_{x}$ for $(x, t) \in[0,1] \times[\tau, T)$. For $x=1$, we get

$$
u_{t}(1, t) \leq-\delta \beta u^{-2 \beta-1}(1, t) .
$$

Integrating for $t$ from $t$ to $T$, we obtain

$$
u(1, t) \geq C_{1}(T-t)^{1 / 2(\beta+1)},
$$

where $C_{1}=(2 \delta \beta(\beta+1))^{1 / 2(\beta+1)}$.

Theorem 11. If $u_{0}$ satisfies (1.2) - (1.3) and (4.1), then there exists a positive constant $C_{2}$ such that

$$
u(1, t) \leq C_{2}(T-t)^{1 / 2(\beta+1)},
$$

for $t$ sufficiently close to $T$. 
Proof. Define $J(x, t)=u_{x}+x u^{-\beta}$ in $[0,1] \times[0, T)$. Then, $J(x, t)$ satisfies

$$
\begin{aligned}
& J_{t}-J_{x x}=\left[\alpha(1-u)^{-\alpha-1}+2 \beta u^{-\beta-1}\right] u_{x} \\
& -\beta x u^{-\beta-1}(1-u)^{\alpha}-\beta(\beta+1) x u^{-\beta-2} u_{x}^{2} .
\end{aligned}
$$

Since $u_{x}<0$, then $J(x, t)$ cannot attain a positive interior maximum. On the other hand, $J(x, 0) \leq 0$ by (4.1) and

$$
J(0, t)=0, J(1, t)=0,
$$

for $t \in(0, T)$. From the maximum principle, we obtain that $J(x, t) \leq 0$ for $(x, t) \in[0,1] \times[0, T)$. Therefore,

$$
J_{x}(1, t)=\lim _{h \rightarrow 0^{+}} \frac{J(1, t)-J(1-h, t)}{h}=\lim _{h \rightarrow 0^{+}} \frac{-J(1-h, t)}{h} \geq 0 .
$$

We get

$$
\begin{aligned}
J_{x}(1, t) & =u_{x x}(1, t)+u^{-\beta}(1, t)+\beta u^{-2 \beta-1}(1, t) \\
& =u_{t}(1, t)-(1-u(1, t))^{-\alpha}+u^{-\beta}(1, t)+\beta u^{-2 \beta-1}(1, t) \geq 0
\end{aligned}
$$

and

$$
u_{t}(1, t) \geq-(\beta+1) u^{-2 \beta-1}(1, t) .
$$

Integrating for $t$ from 0 to $T$, we get

$$
u(1, t) \leq C_{2}(T-t)^{1 / 2(\beta+1)}
$$

where $C_{2}=\left(2(\beta+1)^{2}\right)^{1 / 2(\beta+1)}$.

Corollary 12. Assume the initial datum $u_{0}$ satisfies (1.2) - (1.3). From Theorem 10 and Theorem 11, near the quenching time $T$, the solution $u(x, t)$ to problem (1.1) has the following quenching rate estimate:

$$
u(1, t) \sim(T-t)^{1 / 2(\beta+1)} .
$$

Remark 3. By comparing the problem (1.1) with (1.4), (1.5) for the case of $m=1$ and (1.6), we have from Corollary 12 that although the nonlinearity appears also in the source $(1-u)^{-\alpha}$, the quenching rate still remain the same as that of [2], of [3] and of [9]. Therefore, we say that nonlinearity of source term $(1-u)^{-\alpha}$ has in fact no effect upon the quenching behavior of the problem (1.1) as Remark 4 in [9]. 
Corollary 13. We can calculate a lower bound for the quenching time. From Theorem 11, a lower bound is $u_{0}^{2(\beta+1)}(1) /\left(2(\beta+1)^{2}\right)$ for quenching time $T$.

Remark 4. By comparing problem (1.1) with (1.2), we have from Corollary 13 that, although the nonlinearity appears also in the source $(1-u)^{-p}$, a lower bound for the quenching time smaller (adding extra conditions (1.2) $-(1.3)$ and (4.1)). Namely, $u_{0}^{2(\beta+1)}(1) /\left(2(\beta+1)^{2}\right)<u_{0}^{2(\beta+1)}(1) /(2 \beta(\beta+1))$.

\section{References}

[1] C.W. Chang, Y.H. Hsu and H.T. Liu, Quenching behavior of parabolic systems with localized reaction term, Mathematics and Statistics, 2, No 1 (2014), 48-53.

[2] K. Deng , M. Xu, Quenching for a nonlinear diffusion equation with a singular boundary condition, Z. Angew. Math. Phys., 50 (1999), 574-584.

[3] M. Fila, H.A. Levine, Quenching on the boundary, Nonlinear Anal., 21 (1993), 795-802.

[4] M. Fila, J.-S. Guo, Complete blow-up and incomplete quenching for the heat equation a nonlinear boundary condition, Nonlinear Anal., 48 (2002), 995-1002.

[5] S.C. Fu, J.-S. Guo, J.C. Tsai, Blow up behavior for a semilinear heat equation with a nonlinear boundary condition, Tohoku Math. J., 55 (2003), 565-581.

[6] N. Ozalp and B. Selcuk, The quenching behavior of a nonlinear parabolic equation with a singular boundary condition, Hacettepe Journal of Mathematics and Statistics, 44, No 3 (2015), 615-621.

[7] C. V. Pao, Quasilinear parabolic and elliptic equations with nonlinear boundary conditions, Nonlinear Anal. 66 (2007), 639-662.

[8] B. Selcuk and N. Ozalp, The quenching behavior of a semilinear heat equation with a singular boundary outflux, Quarterly of Appl. Math., 72, No 4 (2014), 747-752. 
[9] Y. Zhi, C. Mu, The quenching behavior of a nonlinear parabolic equation with a nonlinear boundary outflux, Appl. Math.Comput., 184 (2007), 624630. 